Proc. of the 13th Symposium of Magnetic Measurements and Modeling SMMM'2018, Wieliczka, Poland, October 8-10, 2018

\title{
Interpretation of Magnetic Barkhausen Noise Bursts in Low Frequency Measurements
}

\author{
L.F.T. Costa $^{a, *}$, G.J.L. Gerhardt ${ }^{b}$, F.P. Missell ${ }^{b}$ And M.F. DE CAmpos $^{c}$ \\ ${ }^{a}$ CEPEL, PFDF, Divisão de Planejamento e Fomento, Rio de Janeiro, RJ, Brazil \\ ${ }^{b}$ Universidade de Caxias do Sul, Caxias do Sul 95070-560, Brazil \\ ${ }^{c}$ Universidade Federal Fluminense, Volta Redonda 27255-125, Brazil
}

\begin{abstract}
Experimental results allow the identification of three main magnetic Barkhausen noise bursts, each occurring at a different applied field. Magnetostrictive effects can be related to the 1st and 3rd bursts, because closure domain walls are created and/or eliminated. The 2nd burst occurs at the coercive field and it is usually the most intense, and is attributed to domain wall movement. The analysis of the three main bursts gives an important insight on how stress may affect the losses and magnetic Barkhausen noise. A brief review is also presented about the magnetic Barkhausen noise technique.
\end{abstract}

DOI: 10.12693/APhysPolA.136.740

PACS/topics: 75.60.Ej, 75.60.Jk, 75.60.-d

\section{Introduction}

The magnetic Barkhausen noise (MBN) method began in Dresden, with the experiments performed by Heinrich Barkhausen in 1919 [1]. The magnetic Barkhausen noise can provide information about the main dissipative mechanisms in the quasi-static hysteresis. In the present study, measurements performed at $0.5 \mathrm{~Hz}$ are discussed. This situation is near that of quasi-static situation. In the present study, the measurements were performed in toroids, and this means that the exact field where the bursts take place can be determined, and compared with the quasi-static hysteresis.

There are several possible dissipative mechanisms in a hysteresis curve: (i) creation/annihilation of domain walls, (ii) domain wall displacement, (iii) creation/annihilation of closure domain walls with magnetostrictive effects, see Fig. 1 [2, 3], and (iv) domain rotation. In previous investigations, it was noted that domain rotation produces small MBN [4]. MBN provides insight about all these mechanisms. In this study, the discussion will be focused on how to relate MBN bursts and the hysteresis curve.

In the first part of the paper, it is described how to identify the three main bursts, which can appear in MBN measurements. In this study, hysteresis, MBN data, and $\mathrm{d} B / \mathrm{d} H$ are presented in the same graph, allowing identification of the 3 main bursts. In the second part of the paper, a discussion is provided to explain the occurrence of controversies in literature, with some conflicting results reported. Since special attention is given to some commonly neglected details, such as the crystallographic texture of the samples, these controversies can be solved.

*corresponding author; e-mail: marcosflavio@id.uff.br

\section{Experiment}

The samples evaluated in the present study are 1045 or 1020 steels, submitted to different heat treatments. All the samples were austenitized at $910^{\circ} \mathrm{C}\left( \pm 10^{\circ} \mathrm{C}\right)$. After austenitization 1045 and 1020 samples were heat treated during $20 \mathrm{~min}$ at the temperature of $700^{\circ} \mathrm{C}$, and then cooled in air. The normalized 1045 sample was austenitizated followed by air cooling. The quenched 1045 sample was cooled in water after austenitization, generating a martensite structure. More information about the samples, their chemical compositions and their microstructures can be found in previous studies $[4,5]$.

The rms Barkhausen envelopes were measured at $0.5 \mathrm{~Hz}$. With increasing frequency, the burst identification is more difficult. Thus, the use of a very low frequency as $0.5 \mathrm{~Hz}$ was necessary. The presented data are the average considering several Barkhausen envelopes. More details about the experimental procedure of hysteresis and MBN measurement were previously reported [4].

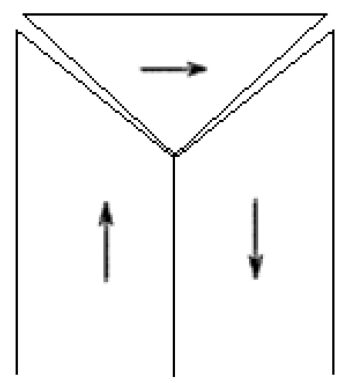

Fig. 1. Due to magnetostrictive effects, there is variation of volume in the direction of magnetization. This gives rise to a misfit along $90^{\circ}$ domain boundaries in iron $[2,3]$. Note that iron is bcc and has 3 easy magnetization axis $\langle 100\rangle$. Thus, both $90^{\circ}$ and $180^{\circ}$ domain walls are possible in iron. 


\section{The three main bursts}

In this study (Figs. 2-5) a clear relation between hysteresis curve, coercivity, and MBN bursts is observed. Each main burst happens at specific field. Three main bursts can be summarized as follows (see the arrows in Figs. 2-5):

1. 1st burst closure domain formation (CDF) at applied field $H$ near 0 .

2. 2nd burst domain wall movement (DWM) for applied field near the coercive field.

3. 3rd burst closure domain annihilation (CDA) at higher applied field.

As magnetostrictive effects strongly affect closure domain walls, then the position and height of the bursts can be altered by applied stresses. In Figs. 2-5, the red curve denotes $\mathrm{d} B / \mathrm{d} H$ curves. Note the difference between the blue curves $(\mathrm{MBN})$ and $\mathrm{d} B / \mathrm{d} H$. The main burst is due to $180^{\circ}$ domain wall movement and appears exactly at the coercive field (except for Fig. 3, which is a sample with martensite). As the main burst is very strong, the burst due to domain wall formation is difficult to detect. If closure domain wall elimination produces a burst, then it is also expected that creation of closure domain walls also generates a burst.

Figure 1 is very important to understand CDF and CDA. The magnetostrictive effects can strongly affect CDF and CDA. Thus, residual stress can be assessed with MBN measurements. In other words, stresses can affect peak intensity and peak position of MBN. Summarizing, there is a clear relationship between $\mathrm{d} B / \mathrm{d} H$ curves and MBN. Three different events were identified, see Eq. (1):

$$
\begin{aligned}
V_{t} & =\frac{\rho}{G}\left[m_{\mathrm{CDF}}\left(h_{\mathrm{CDF}}\right) h_{\mathrm{CDF}}+m_{\mathrm{DWM}}\left(h_{\mathrm{DWM}}\right) h_{\mathrm{DWM}}\right. \\
& \left.+m_{\mathrm{CDA}}\left(h_{\mathrm{CDA}}\right) h_{\mathrm{CDA}}\right],
\end{aligned}
$$

where $V_{t}$ is voltage at a time $t, \rho$ is resistivity, $m_{\mathrm{CDF}}\left(h_{\mathrm{CDF}}\right) h_{\mathrm{CDF}}$ is the contribution of $90^{\circ}$ domain formation, and $m_{\mathrm{DWM}}\left(h_{\mathrm{DWM}}\right) h_{\mathrm{DWM}}$ is the contribution of $180^{\circ}$ domain walls movement to the MBN signal, $m_{\mathrm{CDA}}\left(h_{\mathrm{CDA}}\right) h_{\mathrm{CDA}}$ is the contribution of $90^{\circ}$ domain annihilation. $m_{\mathrm{CDA}}$ is the number of $\mathrm{MBN}$ jumps due to the $90^{\circ}$ domain annihilation at an applied field $h_{\mathrm{CDA}}, m_{\mathrm{DWM}}$ is the number of MBN jumps generated by $180^{\circ}$ domain walls at an applied field $h_{\mathrm{DWM}}$, and this field is approximately the coercive field, $m_{\mathrm{CDF}}$ is the number of MBN jumps produced by the $90^{\circ}$ domain formation at an applied field $h_{\mathrm{CDF}}$. Equation (1) is also named the model of the three Gaussians.

MBN gives information about a $\mathrm{d} B / \mathrm{d} t$ (in other words, it is detected by a voltage $V$ ). Each system uses different filters, and different frequencies of measurement, under different applied fields. Thus, it is difficult to compare the results reported in literature, since different experimental setups can be used [6]. The MBN can be

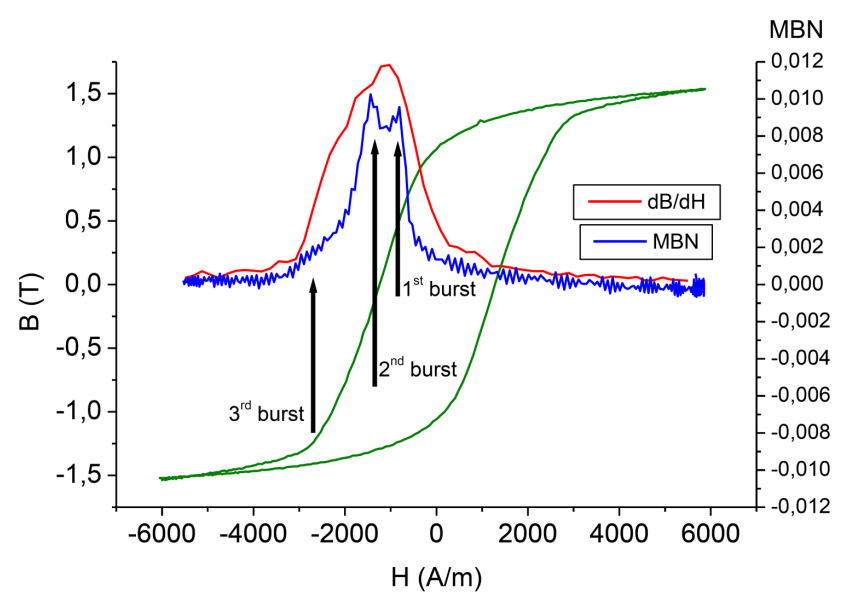

Fig. 2. Sample 1045 steel - normalized. The arrows indicate the three main bursts, $f=0.5 \mathrm{~Hz}$. The MBN scale is relative.

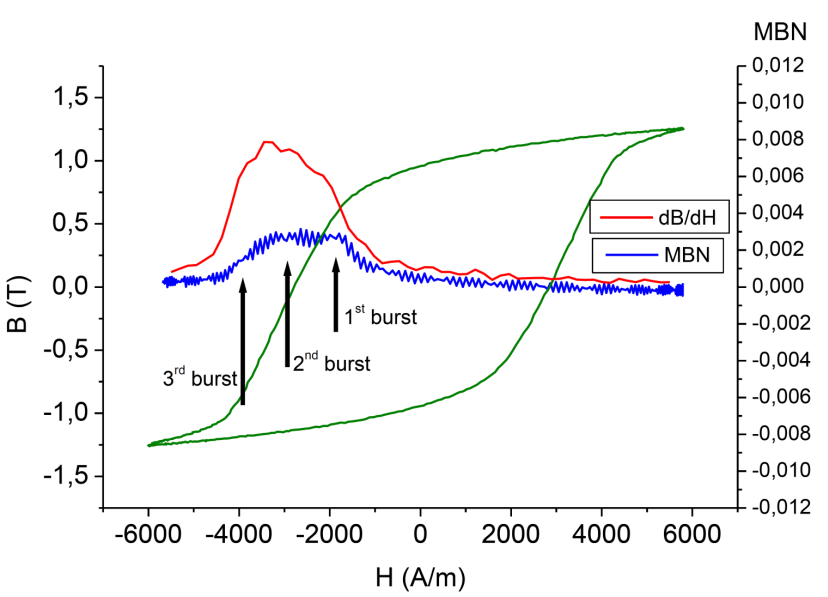

Fig. 3. Sample 1045 steel — quenched. The arrows indicate the three main bursts, $f=0.5 \mathrm{~Hz}$. The MBN scale is relative. Note that for this sample the 2nd burst (due to domain wall movement) is almost null or not observed.

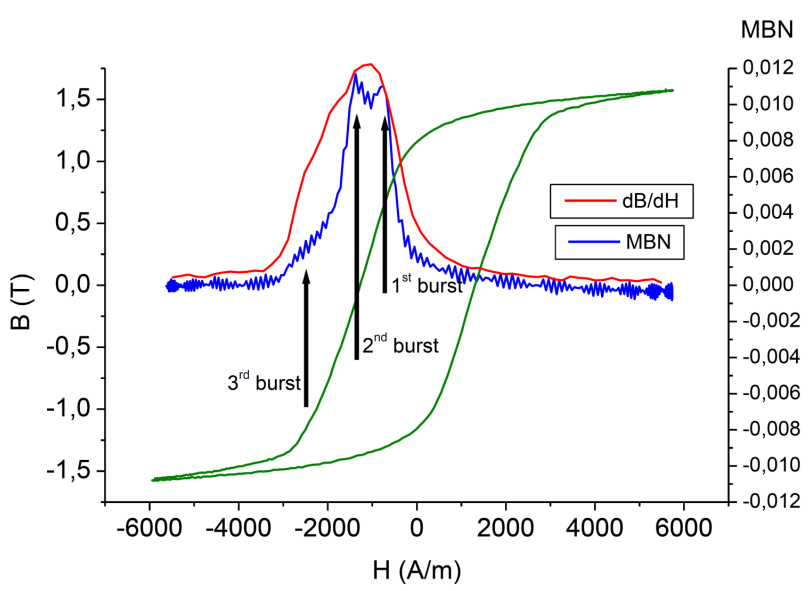

Fig. 4. Sample 1045 steel - heat treated at $700^{\circ} \mathrm{C}$. The arrows indicate the three main bursts, $f=0.5 \mathrm{~Hz}$. The MBN scale is relative. 


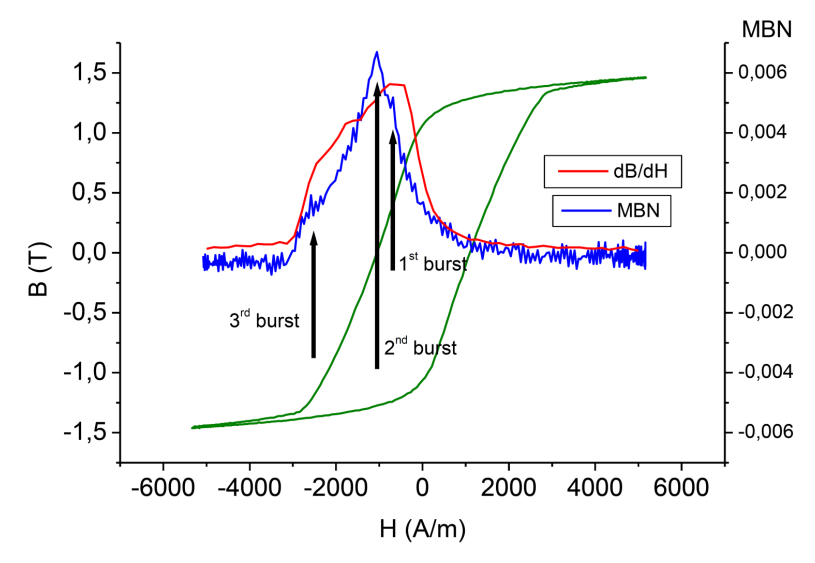

Fig. 5. Sample 1020 steel - heat treated at $700^{\circ} \mathrm{C}$. The arrows indicate the three main bursts, $f=0.5 \mathrm{~Hz}$. The MBN scale is relative.

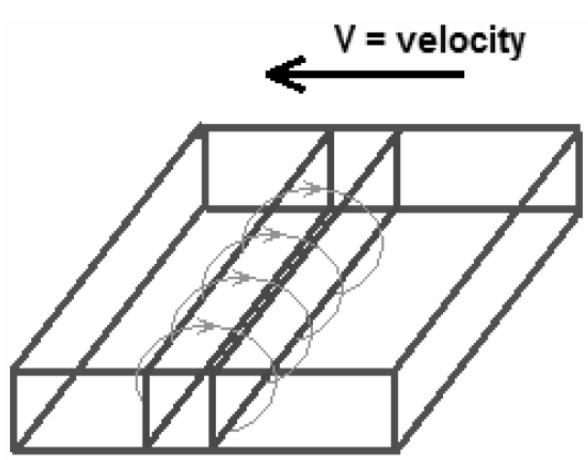

Fig. 6. Illustration of micro eddy currents around one $180^{\circ}$ domain wall, which is moving at a velocity $\boldsymbol{v}$.

attributed in most parts to eddy currents, and the paper of Williams et al. [7], which is the basis for the Pry and Bean model [8] is also the basis for MBN interpretation. The MBN voltage $V$ is related to the speed of the domain wall under to a given applied field, with a damping coefficient $G$, see Fig. 6 .

If the magnetic reversal takes place by rotation, as in nanocrystalline materials $[9,10]$ almost no burst is emitted, since as shown in Fig. 6, the micro eddy currents surrounding domain walls are the main responsible factors for the MBN signal. Thus, for single domain size nanocrystalline materials, a high hysteresis losses and small anomalous loss is observed and only small burst of $\mathrm{MBN}$ is detected $[9,10]$. As can be seen in Fig. 3, martensite generates very small signal, because it is a nanocrystalline structure $[9,10]$. Thus, MBN provides a better understanding of the dissipative mechanisms. The 2nd burst, which is attributed to domain wall movement (see Fig. 6) is not observed in Fig. 3, and the reason is that the reversal of magnetization is due to domain rotation (and not due to domain wall movement).

The typical method for introducing plastic deformation in the samples is cold rolling, which also decreases the sample thickness [11]. Stress-strain tests also reduce the sample thickness. In these cases, it is difficult to separate the effects of thickness and deformation in MBN.

In many cases only 1 peak (interpreted as two or three Gaussian distributions) is observed in MBN measurements [11]. This is because the Gaussian distributions superimpose themselves. When stress is applied, 2 different peaks may be observed instead of one. Thus, in some samples, 2 peaks can be observed, and this is due to applied stress or residual stress.

\section{Interpretation of the $\mathrm{MBN}$ results}

There are many experimental factors affecting MBN. A very important detail is that, for comparative studies, samples should have same volume and geometry. Samples with larger volume will have more pronounced MBN signal. This is a clear scale effect: when the volume increases, the number of domain walls increases. Thus, the samples need to have the same thickness when a series of different samples are compared.

Problems of reproducibility of experimental results may happen. A significant reason is that because different choices are made when performing the MBN experiment in different laboratories. Many authors prefer the use of the RMS - root mean square - of MBN. In the MBN analysis, a field is chosen, as well as a frequency. Typical frequencies are $5 \mathrm{~Hz}$ or $10 \mathrm{~Hz}$. The RMS of MBN will depend significantly on the applied field. If the applied field is less than the coercive field, the signal can be small. Most of the MBN signal happens at near the coercive field, as can be seen in Figs. 2-5.

Another problem in MBN is that effects of microstructure and stress are added. Texture can affect residual stresses [12]. For all measurements of residual stress by X-ray diffraction, the texture should be first evaluated. All specialist on residual stress should first be a specialist on texture. Many researchers fail to correctly interpret their MBN results because they do not have a background on texture and crystallography.

As can be seen in Eq. (2), the elastic stress modifies the anisotropy. The stress effect can be interpreted as a kind of correction for the magnetocrystalline anisotropy energy term. According to [13] the energy $E$ for bcc iron is given as:

$$
\begin{aligned}
E & =K_{1}\left(\alpha_{1}^{2} \alpha_{2}^{2}+\alpha_{2}^{2} \alpha_{3}^{2}+\alpha_{3}^{2} \alpha_{1}^{2}\right) \\
& -\frac{3}{2} \lambda_{100} \sigma\left(\alpha_{1}^{2} \gamma_{1}^{2}+\alpha_{2}^{2} \gamma_{2}^{2}+\alpha_{3}^{2} \gamma_{3}^{2}\right) \\
& -3 \lambda_{111} \sigma\left(\alpha_{1} \alpha_{2} \gamma_{1} \gamma_{2}+\alpha_{2} \alpha_{3} \gamma_{2} \gamma_{3}+\alpha_{3} \alpha_{1} \gamma_{3} \gamma_{1}\right),
\end{aligned}
$$

where $\sigma$ is elastic stress, $\lambda_{100}$ and $\lambda_{l 11}$ are the saturation magnetostriction terms, with strain measured in the $\langle 100\rangle$ and $\langle 111\rangle$ directions. $\gamma_{1}, \gamma_{2}$, and $\gamma_{3}$ are the direction cosines of stress, and $\alpha_{1}, \alpha_{2}$ and $\alpha_{3}$ are the direction cosines of the magnetization of saturation $-M_{S} . K_{1}$ is the first constant of magnetocrystalline anisotropy. 
As can be seen in Eq. (2), the direction of $M_{S}$ changes when some elastic stress $\sigma$ is applied. More importantly, Eq. (2) shows that depending on the crystal orientation, the applied stress can have opposite effects. This probably explains many controversial results in literature, where the authors rarely asses the texture of the material when using MBN.

In other words, the effect of applied stress on a single crystal can be interpreted as a change on the magnetocrystalline anisotropy constants. This means that stresses can either increase or decrease the coercivity. Thus, samples with different texture can produce different results.

\section{Comparison with other techniques as X-ray diffraction}

The stress state can make the MBN pattern to be altered. It should be emphasized again that the samples need to have same volume and geometry for MBN comparison. Besides, the type of probe can affect the signal [6]. The relevant detail is that stress can affect peak and intensity of magnetic Barkhausen noise. Elastic stresses affect the signal, and most of the MBN signal is from the surface [14]. In Eq. (3), $\delta$ is the depth of penetration (also called skin depth), $\rho$ is resistivity, and $\mu$ is permeability. Note that by increasing frequency, the signal comes from the region nearest to surface. The permeability of the material also affects the skin depth. For example, plastic deformation decreases the permeability significantly

$$
\delta=\sqrt{\frac{\rho}{\pi \mu f}}
$$

In X-ray diffraction, most of the signal is also from the surface. In steels most of the X-ray diffraction detected signal comes from first $10 \mu \mathrm{m}$, and over $90 \%$ from the first $20 \mu \mathrm{m}$ (under the typical copper $K_{\alpha}$ radiation). The $G_{x}$ fraction of the intensity of the measured signal is given by $G_{x}=1-\exp \left(-2 \mu_{\mathrm{abs}} x_{p} \sin \theta\right)$, where $\mu_{\mathrm{abs}}$ is the linear absorption coefficient, $x_{p}$ is thickness, and $\theta$ is the Bragg angle. $\mu_{\mathrm{abs}}$ is function of the chemical composition of the sample and also of the wavelength of the incident radiation.

The macrostresses can be accounted with a common $\mathrm{X}$-ray diffraction equipment [14], since only a uniaxial stress is applied. A rough estimation of elastic stress in the direction of the measurement can be done by means of the expression $\sigma=\varepsilon E_{\text {Young }} / \nu$ with $\varepsilon=\left(d-d_{0}\right) / d$. For steels, $E_{\text {Young }}=170 \mathrm{GPa}$ and $\nu=0.3$ [14]. $d$ is interplanar distance, $\nu$ is Poisson coefficient, $E_{\text {Young }}$ is Young modulus, $\varepsilon$ is deformation. This is a method that can be used in normal X-ray diffraction equipments, with the Bragg-Brentano geometry.

In recent studies, MBN data has been compared with X-ray diffraction and hole drilling method $[15,16]$. In these experiments, the hole drilling method was performed with a hole with $2 \mathrm{~mm}$ of depth, but with extensometer placed at the surface $[15,16]$. Thus, some variation of presented results $[15,16]$ should be due to the fact that these different techniques (MBN, X-ray diffraction, and hole drilling) assess stress at different depths. MBN is an indirect method, and always need to compared with direct methods, as X-ray diffraction and hole drilling. Besides, MBN signal includes both stress and microstructure effects. This means that the analysis of the MBN signal is not an easy task.

It is also relevant to distinguish the macrostresses (i.e., the residual stresses) from the microstresses, which are due to dislocations. The microstresses can be assessed from X-ray diffraction data [17-19]. Dislocations present stress field of high order (near $1 \mathrm{GPa}$ ) [20]. As consequence of plastic deformation, coercivity increases considerably and the permeability is significantly reduced. This happens even with very small plastic deformation [17-19].

Last, but not least, it should be reminded that in static equilibrium $\Sigma F=0$ and $\Sigma M=0$ (i.e. the sum of forces and momentum are null), where $F$ is force and $M$ is momentum. Thus, if the sample is cut, then all the residual stresses are redistributed. This detail is very relevant: for evaluating residual stresses the samples never can be cut and the same sample should be used for X-ray diffraction and for MBN experiments; otherwise different results will be obtained.

\section{Conclusions}

MBN is a technique able to give profound insight on the magnetization processes. However, MBN needs to be used with extreme care. Most of the MBN signal usually comes from $180^{\circ}$ domain wall movement.

There is a close relationship between $\mathrm{d} B / \mathrm{d} H$ curves and magnetic Barkhausen noise. The three main observed bursts can be summarized as follows: (i) CDF at applied field $\mathrm{H}$ near 0. (ii) DWM for applied field near the coercive field. (iii) CDA at higher applied field.

MBN is very sensitive, then even very small variations in volume can affect the MBN signal. Samples need to have the same volume and thickness for comparison.

\section{Acknowledgments}

Work supported in part by FAPERJ (Fundação Carlos Chagas Filho de Amparo à Pesquisa do Estado do Rio de Janeiro) and CNPq (Conselho Nacional de Desenvolvimento Científico e Tecnológico)

\section{References}

[1] H. Barkhausen, Phys. Zeitschr. 20, 401 (1919).

[2] C. Kittel, Rev. Mod. Phys. 21, 541 (1949).

[3] W.F. Hosford, Iron and Steel, Cambridge University Press, 2012.

[4] L.F.T. Costa, M.F.D. Campos, G.J.L. Gerhardt, F.P. Missell, IEEE Trans. Magn. 50, 2001504 (2014). 
[5] L.F.T. Costa, F. Girotto, R. Baiotto, G. Gerhardt, M.F. de Campos, F.P. Missell, J. Phys. Conf. Series 303, 012029 (2011).

[6] J.C. Sánchez, M.F. de Campos, L.R. Padovese, J. Nondestruct. Evaluat. 36, 66 (2017).

[7] H.J. Williams, W. Shockley, C. Kittel, Phys. Rev. 80, 1090 (1950).

[8] R.H. Pry, C.P. Bean, J. Appl. Phys. 29, 532 (1958).

[9] M.F. de Campos, F.A. Franco, R. Santos, F.S. da Silva, S.B. Ribeiro, J.F.C. Lins, L.R. Padovese, J. Phys. Conf. Series 303, 012030 (2011).

[10] E.F. Monlevade, M.F. de Campos, F.A. Franco, J. Capo-Sanchez, H. Goldenstein, L.R. Padovese, IEEE Trans. Magn. 48, 1465 (2012).

[11] M.F. de Campos, F.R.F. Da Silva, J.F.C. Lins, E.F. Monlevade, M.A. Campos, et al., IEEE Trans. Magn. 49, 1305 (2013).

[12] V. Hauk, Structural and Residual Stress Analysis by Nondestructive Methods: Evaluation - Application - Assessment, Elsevier, 1997.

[13] B.D. Cullity, C.D. Graham, Introduction to Magnetic Materials, 2nd ed., Wiley, 2008.
[14] J.C. Sánchez, M.F. de Campos, L.R. Padovese, J. Magn. Magn. Mater. 324, 11 (2012).

[15] S.R. Correa, M.F. de Campos, C.J. Marcelo, J.A. de Castro, M.C. Fonseca, T.C. Chuvas, M.A. Campos, L.R. Padovese, Mater. Sci. Forum 869, 556 (2016).

[16] S.R. Correa, M.F. de Campos, C.J. Marcelo, J.A. de Castro, M.C. Fonseca, T.C. Chuvas, M.A. Campos, L.R. Padovese, Mater. Sci. Forum 869, 567 (2016).

[17] M.F. de Campos, M.J. Sablik, F.J.G. Landgraf, T.K. Hirsch, R. Machado, R. Magnabosco, et al., J. Magn. Magn. Mater. 320, e377 (2008).

[18] P.P. de C. Antonio, M.F. de Campos, F.M.D.S. Dias, M.A. Campos, J. Capo-Sanchez, L. R. Padovese, IEEE Trans. Magn. 50, 2001704 (2014).

[19] D.L. Rodrigues, Jr., J.R.F. Silveira, G.J.L. Gerhardt, F.P. Missell, F.J.G. Landgraf, R. Machado, M.F. de Campos, IEEE Trans. Magn. 48, 1425 (2012).

[20] M.F. de Campos, Mater. Sci. Forum 727, 157 (2012). 